\title{
Deep phenotyping platform for microscopic plant-pathogen interactions
}

1 Stefanie Lück ${ }^{1}$ and Dimitar Douchkov ${ }^{1}$

$2 \quad{ }^{1}$ Leibniz Institute of Plant Genetics and Crop Plant Research (IPK), D-06466 Seeland, Germany

3

4 Author for correspondence:

5 Dimitar Douchkov

6 Tel. +493948252825

7 Email: douchkov@ipk-gatersleben.de

9 Keywords: automated microscopy, barley, BluVision, Deep learning, microphenomics, 10 neuronal networks, pathogens, plant, powdery mildew, wheat.

12 Word counts:

13 Total main body: 5684

14 . Summary: 200

15 . Introduction: 931

16 . Material and Methods: 1205

17 . Results: 2690

18 . Discussion: 657

19 Number of figures: 11 (color)

20 Tables: 7

21 Supporting information: 1 figure, 4 tables 


\section{Summary}

- The initial phases of plant-pathogen interactions are critical since they are often decisive for the successful infection. However, these early stages of interaction are typically microscopic, making it challenging to study on a large scale.

- For this reason, using the powdery mildew fungi of cereals as a model, we have developed an automated microscopy pipeline coupled with deep learning-based image analysis for the high-throughput phenotyping of plant-pathogen interactions.

- The system can quantify fungal microcolony count and density, the precise area of the secondary hyphae of each colony, and different morphological parameters. Moreover, the high throughput and sensitivity allow quantifying rare microscopic phenotypes in a large sample size. One of these phenotypes is the cryptic infection of non-adapted pathogens, marking the hidden transition stages of pathogen adaptation and breaking the nonhost barrier. Thus, our tool opens the nonhost resistance phenomenon to genetics and genomics studies.

- We have developed an open-source high-throughput automated microscopy system for phenotyping the initial stages of plant-pathogen interactions, extendable to other microscopic phenotypes and hardware platforms. Furthermore, we have validated the system's performance in disease resistance screens of genetically diverse barley material and performed Genome-wide associations scans (GWAS), discovering several resistanceassociated loci, including conferring nonhost resistance.

\section{Introduction}

44 Public authorities and society, particularly in Europe, mostly agree about an agroecological

45 transition toward a chemical pesticide-free and GMO-free agriculture. However, this ambitious

46 aim might be challenged by increased outbreaks of new aggressive pathogens promoted by global

47 trade, monocultures, and climatic changes. As high as $40 \%$ of global crop production is lost due

48 to pests and diseases, regardless of the extensive use of pesticides (FAO, 2020). Therefore, reduced

49 chemical pesticide use without compensating measures will threaten global food safety to an

50 unacceptable level. One of the most sustainable and environmentally friendly alternatives to

51 chemical pesticides is employing the natural disease resistance of plants. This approach was 
52 successfully used in the long history of crop breeding. Still, to meet the new challenges, the plant

53 breeders need to discover new disease resistance sources by digging deep into the genetic diversity

54 stored in the gene banks and germplasm collections worldwide by using more sensitive

55 phenotyping tools capable of discovering quantitative trait loci (QTLs) even with minimal effects

56 and low allele frequency.

57 The scientific community has identified this need and initiated precise and high-throughput

58 phenotyping tools to establish a new scientific discipline called phenomics. However, most of

59 these efforts were aimed at phenotyping on a larger object level, such as whole plants and canopy,

60 with an insufficient spatial resolution for detailed studies of the typically microscopic plant-

61 pathogen interactions. To contribute to this bottleneck's alleviation, we started developing a highly

62 automated phenotyping platform to cover the subcellular, tissue, and organ level of phenotyping.

63 The system for organ-level phenotyping on a macroscopic scale called Macrobot, and the

64 corresponding software framework (BluVision Macro), were published previously (Lück et al.,

65 2020; Lueck et al., 2020). This article is focused on the high-throughput microscopic system for

66 phenotyping on the cellular and subcellular level, named BluVision Micro.

67 The primary aim of the BluVision framework is the phenotyping of plant-pathogen interactions on

68 microscopic and macroscopic levels. As a model for the development was selected, the well-

69 established system of the powdery mildew fungus Blumeria graminis as a pathogen of barley and

70 wheat (Panstruga and Dodds, 2009; Spanu and Kamper, 2010; Douchkov et al., 2014).

71 B. graminis is the only species of the ascomycete genus Blumeria, the order of Erysiphales. They

72 are causing powdery mildew diseases on many different grass species. All Blumeria graminis are

73 obligate parasites with typically extremely specific host-specialization forms, called formae

74 speciales (ff.spp.), e.g., B. graminis f. sp. tritici (wheat powdery mildew, Bgt), and the B. graminis

75 f. sp. hordei (barley powdery mildew, Bgh) (Wyand and Brown, 2003). Typically the plants are

76 entirely immune against the non-adapted pathogens, e.g., barley is immune to Bgt and wheat to

77 Bgh. However, some plant genotypes may allow microscopic growth of non-adapted pathogens,

78 known as cryptic infection (Romero et al., 2018; Bourras et al., 2019; Bettgenhaeuser et al., 2021).

79 The barley/wheat - powdery mildew model provides several advantages to the researchers: the

80 fungus growth is fast and highly synchronized, the majority of the fungal biomass is located on

81 the leaf surface, with straightforward to observe structures. Furthermore, the fungus interacts only 
82 with the uppermost layer of plant leaf cells, the epidermis, via a specialized intracellular feeding 83 organ called a haustorium (Huckelhoven and Panstruga, 2011). This system of reduced complexity 84 provides an excellent environment for studying plant-pathogen interactions on a microscopic scale.

85 However, full-size and multilevel microscopy images of large objects, such as leaf segments, are 86 typically significant portions of complex data that were only very limitedly accessible with automated image analysis methods until recently. The situation improved dramatically with the coming of age of machine learning (ML) methods that use analytical models to identify patterns and make decisions with minimal human intervention (Mitchell, 1997; Voulodimos et al., 2018). There are two main approaches to ML - supervised learning from pre-labeled data (Russell, 2010) and unsupervised learning from unlabeled data (Hinton, 1999). The analysis of images usually includes classification and segmentation steps. The image classification uses features (variables) from images that help classify the objects. The image segmentation assigns labels to the individual pixels, groups them into subgroups (image objects), and subtracts them from the background (Stockman and Shapiro, 2001). Choosing meaningful classification features (feature engineering) (Zheng and Casari, 2018) can be crucial for the success of image analysis. This work compares two main methods - selecting features by human decision (handcrafted features) and automatically extracting features using a convolutional neural network (CNN). CNN can automatically select many features, which leads to more robust prediction models. The downside of the CNNs is the requirement of large training datasets, where predictive models like Random forest (RF) with carefully selected handcrafted features show satisfying results even on small training sets (Lin et

102 al., 2020). The optimal approach depends on the specific application and typically would require 103 preliminary testing of different methods.

104 Here we present the BluVision Micro system dedicated to phenotyping the initial stages of plant105 pathogen interactions using high-throughput automated microscopy and computer vision methods 106 for localization and quantification of microscopic fungal structures. Unlike the macroscopic 107 systems that typically quantify the disease's visible symptoms, the BluVision Micro delivers 108 precise information about the pathogen behavior, the host's early response to the pathogen attack, 109 and the fungus's biomass and growth, virtually eliminating the environment's effects. 


\section{Related work}

113 The first software development for segmentation and quantifying secondary hyphae of $B$. graminis

114 f. sp. hordein (barley powdery mildew) was the HyphArea Tool (Seiffert and Schweizer, 2005;

115 Baum et al., 2011). The software was developed in Python 2. It is based on a histogram-based

116 threshold for hyphae segmentation and a shape descriptor for classifying the regions of interest

117 (ROI).

\section{$4 \quad$ Material and methods}

\subsection{Plant and fungal material}

120 Barley cv. Golden Promise and cv. Morex, and wheat cv. Kanzler were grown in $12 \mathrm{~cm}$ pots with 121 IPK-soil substrate. The plants were incubated in a plant growth cabinet (Sanyo/Panasonic MLR122 352H-PE Versatile Environmental Test Chamber, white LED upgrade; Panasonic Healthcare Co., 123 Ltd.) at controlled conditions (dark period of $8 \mathrm{~h}$, light period of $16 \mathrm{~h}, 20^{\circ} \mathrm{C}$ and $60 \mathrm{RH} \%$ ) for 7 days 124 or 14 days. The second leaves were harvested and mounted on 1\% water agar (Phyto agar, 125 Duchefa, The Netherlands) plates supplemented by $20 \mathrm{mg} / \mathrm{L}$ benzimidazole as a senescence 126 inhibitor. The barley leaf segments were inoculated with the Bgh isolate $\mathrm{CH} 4.8$, and the wheat leaf 127 segments were inoculated with Bgt isolate FAL92315 at approximately 5 spores $/ \mathrm{mm}^{2}$ in an 128 inoculation tower. The fungus was stopped at 36-96 hours after inoculation (hai) by incubating the 129 leave segments in a clearing solution (7 mL 96\% Ethanol and $1 \mathrm{~mL}$ Acetic acid) for 48 hours at 130 room temperature. After that, the fungal colonies were stained with Coomassie staining solution 131 (0.3\% Coomassie R250, 7.5\% (w/v) trichloroacetic acid, and 50\% (v/v) methanol) for 5 minutes 132 and then washed several times with water. The prepared samples were mounted on microscope 133 slides with $50 \%$ glycerol to avoid drying the leaves during image acquisition.

134 The material of the barley core collection of genotypes was grown, collected, and inoculated as 135 described in (Lück et al., 2020). In brief, the plants were grown in 24-well seedling trays, ten plants 136 of the same genotype per well, in a climatized greenhouse for 14 days. Leaf fragments from the 137 second leaf were harvested and mounted on standard 4-well microtiter plates, filled with $1 \%$ water 138 agar supplemented by $20 \mathrm{mg} / \mathrm{L}$ benzimidazole. The leaf fragments were inoculated, incubated, and 139 stained as described above. 


\subsection{Image acquisition and analysis hardware}

141 The microscopy image data was acquired on a commercial Zeiss AxioScan.Z1 high-performance

142 microscopy slide scanner and ZEN 3.0 (blue edition) software (Carl Zeiss AG). The imaging was

143 done in a bright field configuration with a Hitachi HV-F202SCL camera (3 CCD 1/1.8" progressive

144 scan color sensor with 1600x1200 effective pixels and 24-bit color depth), 1x camera adapter. As

145 scanning objective typically was used an EC Plan-Neofluar 5x/0.16 M27 with 0.16 NA (numerical

146 aperture) that provided a large depth of field (DoF), which was particularly advantageous for

147 scanning very thick and uneven objects as whole-leaf fragments and helped reduce the Z-stack

148 levels to only five by keeping the most fungal structure focus. The acquired image data was stored

149 in a CZI file container that combines all relevant image and meta information in one file. The

150 image data were analyzed on a Windows 10 Enterprise server with a double Intel Xeon ${ }^{\mathrm{TM}}$ E5-2695

151 processor with 36 physical cores and 512 GB RAM, allowing nearly real-time analysis if required.

152 The macroscopic image data were acquired six days after infection, as described in (Lück et al.,

153 2020). Monochrome images in all illumination modes were acquired separately and stored in 16-

154 bit TIFF image files.

\section{$155 \quad 4.3$ Software implementation}

156 The software BluVision Micro and all experiments were implemented in Python 3.6 under 157 Windows 10 operating system. The following free Python libraries were used for development: 158 OpenCV-Python, NumPy, Pandas, Keras, Tensorflow, czifile, skimage, mahotas, joblib and Scikit159 learn. Training of the CNN model was done on NVIDIA TITAN X GPU with Keras 2.3.1 and 160 Tensorflow 2.1.0 backend, and training time of about 20.000 images per hour on an Intel ${ }^{\circledR}$ Core $^{\mathrm{TM}}$ 161 i7-9700 CPU 3.00 GHz with 64-Bit Windows 10 operation system.

162 The software is implemented as a two-step command-line tool with separated image processing 163 and data analysis, allowing curation of the intermediate results without rerunning the entire 164 analysis. In addition, the images processing can be parallelized, depending on the installed 165 computer memory. 


\subsection{Barley Genotyping}

169 Two hundred barley accessions from the barley collection of the Federal ex-situ Genebank in

170 Gatersleben, selected for maximized genetic diversity, were genotyped by using whole-genome 171 sequencing (WGS) data from Illumina short-read sequencing with 3x genome coverage (Milner et 172 al., 2019), and aligned to the barley MorexV2 reference genome (König et al., 2020; Mascher, 173 2020) (Supplemental Figure S1). A quality filter on 223387147 variants was applied with the 174 Plink 2.0 software as follows: missing values $\leq 0.02$ and minor allele frequency (MAF) $\geq 0.05$. 175 After filtering, 949174 high-quality variants remained and were used in GWAS analysis.

\subsection{Best linear unbiased estimator (BLUE)}

177 To obtain robust and unbiased phenotype means for the individual genotypes from the three 178 independent experiment repetitions, we used the Best linear unbiased estimator (BLUE) 179 (Henderson, 1975; Liu et al., 2008). BLUE were calculated by using the lme4 library for R using 180 the spore inoculation density as fixed factor.

\subsection{Genome-Wide Association Study}

182 GWAS for the seven traits was conducted with a Factored Spectrally Transformed Linear Mixed 183 Model using a kinship (K) matrix provided by the FaST-LMM program (fastlmm 0.5.5) (Lippert 184 et al., 2011; Listgarten et al., 2012). Suggestive threshold (- $\log 10 \mathrm{P} \geq 6.0)$ was calculated based 185 on the formula - $\log 10$ (1/ number of independent SNPs)(Yang et al., 2014) and significance 186 threshold (- $\log 10 \mathrm{P} \geq 8.0)$ for the identification of QTLs was calculated by using the Bonferroni 187 correction method (Hommel, 1988).

\subsection{Phenotype Preprocessing}

189 Six direct phenotypes and one derivative were derived for each leaf sample (Figure 1). The 190 microscopic phenotypes include normalized colony counts at 48 and 96 hours after infection (hai) 191 with the adapted pathogen (Bgh), and 96 hai with the non-adapted fungus (Bgt). In addition, one 192 macroscopic phenotype (infection spread at 168 hai) was included for comparison (Table 1). 


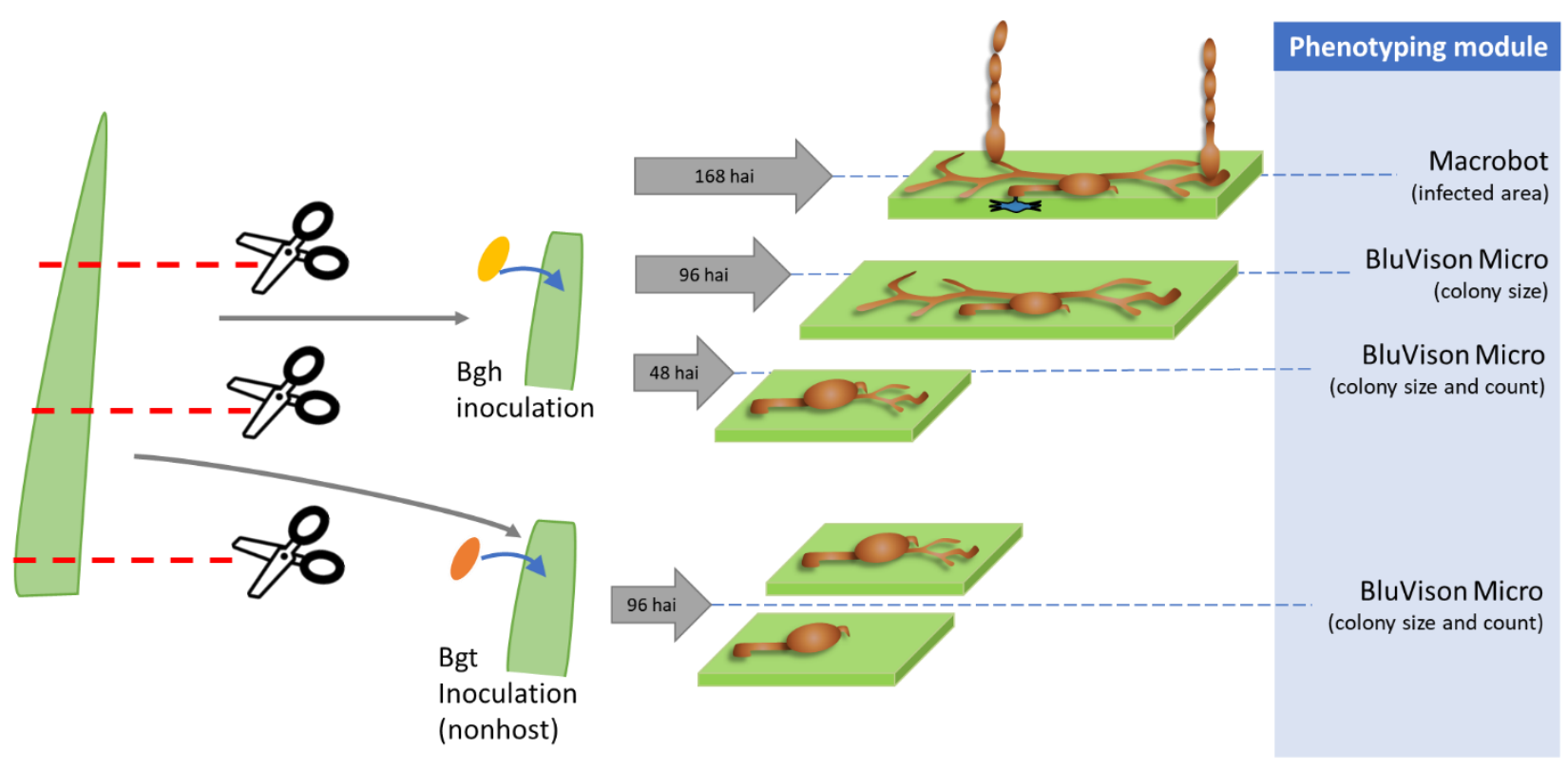

197 Figure 1. Microscopic and macroscopic phenotypes derived from a single leaf. Up to eight barley 198 plants of the same phenotype were grown for 14 days. Two segments from the second leaf of each 199 plant were cut and inoculated with adapted (Bgh) or non-adapted (Bgt) pathogen. Samples for 200 microscopy were collected at 48 and 96 hai.

Table 1. Analyzed phenotypes.

\begin{tabular}{llllll}
\hline Phenotype & Phenotyping module & Scale & Pathogen & Time (hai) & Interaction type \\
\hline Bgh_48hai_counts & BluVision Micro & Micro & Bgh & 48 & host \\
Bgh_48hai_size & BluVision Micro & Micro & Bgh & 48 & host \\
Bgh_96hai_size & BluVision Micro & Micro & Bgh & 96 & host \\
Bgt_96hai_counts & BluVision Micro & Micro & Bgt & 96 & nonhost \\
Bgt_96hai_counts_bin & BluVision Micro & Micro & Bgt & 96 & nonhost \\
Bgh_48-96hai_slope & BluVision Micro & Micro & Bgh & $48-96$ & host \\
Bgh_168hai_area & Macrobot & Macro & Bgh & 168 & host
\end{tabular}

205 For colony mean size per leaf 48 and 96 hours after Bgh inoculation, the colony area was extracted 206 from the segmented images with the OpenCV contourArea() function, and the BLUE was 
calculated from the mean of three experiment repetitions for each barley genotype. The colony

208 sizes at both time points were used to calculate the slope of the growth curve, which was also used 209 as a phenotype in GWAS.

210 In addition to the quantitative phenotype (normalized colony counts) for the non-adapted pathogen 211 (96 hai Bgt), we also calculated a binary qualitative phenotype using a threshold for the normalized 212 colony count of 0.1 . This approach reflects the qualitative nature of the NHR and allows for the 213 identification of major R-genes involved in this complex phenomenon.

214 The macroscopic infection severity was calculated as the percentage of leaf area covered by the 215 powdery mildew colonies 168 hai using the BluVision Macro software (Lueck et al., 2020). A 216 mean of up to 8 technical replicates per accession in an experiment was used to calculate the BLUE 217 values.

\section{Results}

$219 \quad 5.1 \quad$ Image processing

\section{$220 \quad$ 5.1.1 Focus stacking}

221 For finding the optimal focus stacking strategy of the multilevel CZI-images, we have tested five 222 different Z-projection methods included in the Fiji distribution package of ImageJ v1.53 - Average 223 intensity (Khamfongkhruea et al., 2017), Maximum intensity (Sato et al., 1998), Minimum 224 intensity (Hayabuchi et al., 2011), Sum slices, Standard deviation and Median (Figure 2).

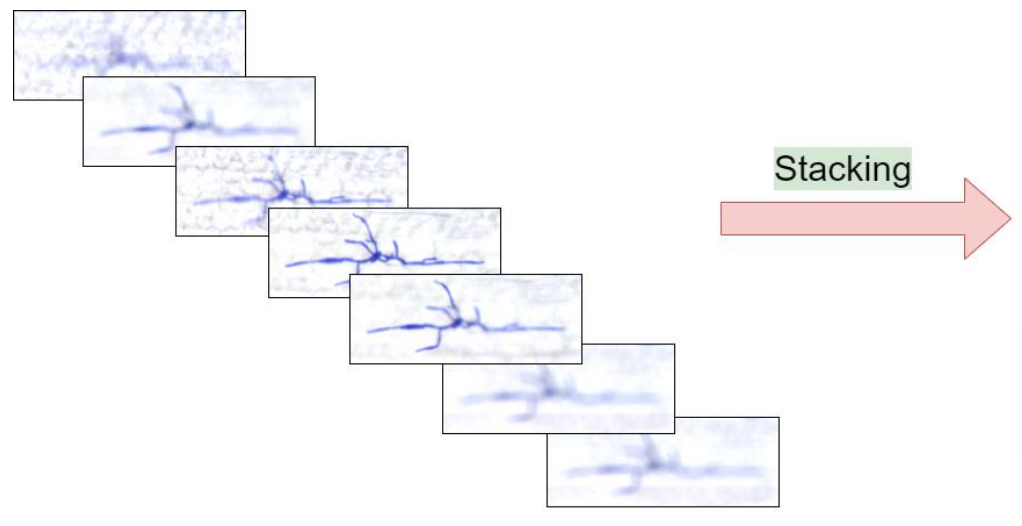

Z-Stack

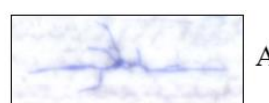

Average intensity $(\mathrm{FM}=0.0690)$

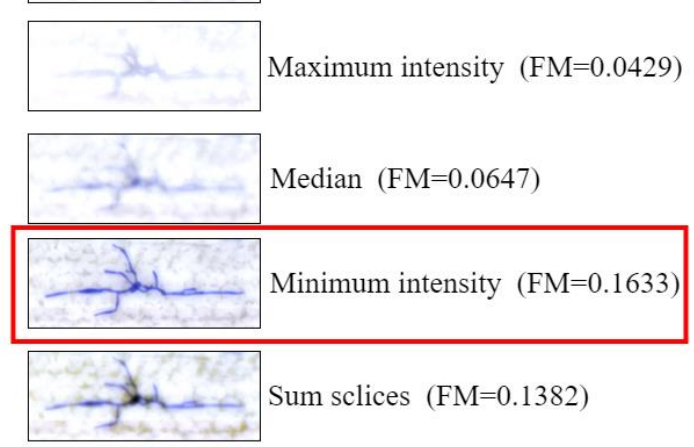

Stacked image

Figure 2. Comparing stacking algorithms. Five stacking algorithms were compared: Average intensity, Maximum intensity, Median, Minimum intensity, Sum slices. The Minimum intensity 228 method achieved the highest quality measure (FM). 
229 Furthermore, for each stacked image, the image Quality Measure (FM) has been computed and 230 compared (Table 2) (De and Masilamani, 2013). The minimum intensity projection method 231 achieved the best FM score in all tested cases and was selected for the image processing pipeline.

233 Table 2. Intensity Z-projection methods compared on two image stacks

234

235

236

237

238

239

240

\begin{tabular}{ccc}
\hline Stack Nr. & Method & FM \\
\hline Stack 1 & average & 0.0449 \\
Stack 1 & maximum intensity & 0.0444 \\
Stack 1 & median & 0.0456 \\
Stack 1 & minimum intensity & 0.0825 \\
Stack 1 & sum slices & 0.0691 \\
Stack 2 & average & 0.0690 \\
Stack 2 & maximum intensity & 0.0429 \\
Stack 2 & median & 0.0647 \\
Stack 2 & minimum intensity & 0.1633 \\
Stack 2 & sum slices & 0.1382 \\
\hline
\end{tabular}

\subsubsection{Colony segmentation}

The fungal colony images were extracted and classified in several steps. A significant challenge was to design a reliable pipeline that tolerates staining quality and background variability without losing too many positive objects.

First, the Z-stacked images were segmented to find the putative ROIs. Then, regions of interest were extracted as a bounding box, and the image was classified into a positive or negative class.

Different common color spaces were tested: HSV, L*a*b, YCbCr, XYZ, AC1C2, YUV, I1I2I3 and YQ1Q2, in combination with different thresholding algorithms: Yen's maximum correlation (Yen et al., 1995), Li's minimum cross-entropy method (Li and Lee, 1993; Li and Tam, 1998; Sezgin and Sankur, 2004), Otsu (Otsu, 1979), Isodata (Ridler and Calvard, 1978), Mean (Glasbey, 1993), Minimum (Prewitt and Mendelsohn, 1966; Glasbey, 1993), Triangle (Zack et al., 1977), Canny edge detector (Canny, 1986) (Table 3). Combining the Q2 channel from the YQ1Q2 color space with Yen's thresholding generated the most reliable results. Using only a single-color channel, we achieved a robust and reliable segmentation method that is insensitive to staining variations and performs well on different sizes of the hyphae (36 to 72 hai). 
252 Table 3. Segmentation methods for colony detection (image of $30000 \times 12000$ pixels containing

253120 colonies). Comparision of the used software libraries, run-time per image and colony 254 segmentation performance.

\begin{tabular}{lcccc}
\hline \multicolumn{1}{c}{ Method } & Library & Time(s) & $\begin{array}{c}\text { Segmented } \\
\text { colonies }\end{array}$ & $\begin{array}{c}\text { Partial } \\
\text { segmented } \\
\text { colonies }\end{array}$ \\
\hline Canny edge detector & OpenCV & 6.5 & 30 & 109 \\
Global thresholding & OpenCV & 0.3 & 44 & 5 \\
Adaptive Thresholding Mean & OpenCV & 2.0 & 87 & 20 \\
Adaptive Thresholding Gaussian & OpenCV & 3.9 & 86 & 5 \\
Otsu's Binarization & OpenCV & 1.4 & 79 & 16 \\
Li Minimum & scikit-image & 112.0 & 4 & 22 \\
Yen thresholding & scikit-image & 81.0 & 16 & 19 \\
\hline
\end{tabular}

255

256 A morphological closing operation was applied to the segmented binary images to close the gaps 257 that may lead to partial object extraction. Finally, a Moore-Neighbour tracing algorithm 258 (Weisstein, 2021) was used to extract the contours of the binary image for colony classification.

\section{$260 \quad 5.2 \quad$ Machine learning}

\section{$261 \quad$ 5.2.1 Training data set}

262 Bgh inoculated barley leaves were Coomassie-stained at 36-72 hai and scanned with the 263 AxioScan.Z1 system. The multilevel images were processed as described above. The putative ROIs 264 were extracted with a bounding box and saved as separate images. The images were manually 265 curated, and about 10000 ROI containing fungal colonies were selected. Another 8000 images 266 without any fungal structures but other objects and artifacts were selected as negative training data. 267 Finally, a small training set with 3200 images per class was extracted from the large training set 268 to study the prediction performance based on the training set size.

269 Both datasets were split randomly into $75 \%$ of the images for training the models and $25 \%$ for 270 validation and evaluation. Since the Convolutional Neuronal Network (CNN) approach requires 271 identical dimensions of the training images, they were resized to $150 \times 350$ pixel, the mean ROI 272 size of the particular data set. 


\section{$273 \quad$ 5.2.2 Classification using handcrafted features}

274 Manual selection of features for building a reliable classifier is still a widely used approach that,

275 in some cases, may outperform more sophisticated methods (Lück et al., 2020). However, the

276 success of this approach strongly depends on the selection of informative and robust features. In

277 our case, of particular importance was to select color- and scale-invariant features because of the

278 high staining intensity- and colony size variation.

279 The contours received after the segmentation step were first filtered using geometrical features

280 (Table 4) to reduce artifacts and non-fungal structures.

Table 4. Object size parameters for filtering colonies from the artifacts. Minimum and maximum 283 thresholds for colonies are indicated.

\begin{tabular}{ccc}
\hline Feature & Minimum pixel values & Minimum pixel values \\
\hline Width & 100 & 1400 \\
Height & 100 & 800 \\
Aspect ratio & 0.5 & 10.0 \\
Area & 1000 & 30000 \\
\hline
\end{tabular}

285 Then, five scale- and color-invariant features (Histogram of oriented Gaussians (Dalal and Triggs, 286 2005), Local binary pattern (Dong-chen and Li, 1990; Wang and He, 1990), Haralick (Haralick et 287 al., 1973), Zernike Moments (Tahmasbi et al., 2011), Parameter-free threshold adjacency statistics 288 (Coelho et al., 2010); Table 5) were extracted with the mahotas and scikit-image library, and a 289 random forest classifier with 80 trees was trained with the two training sets (3 200 and 10000 290 images per class).

Table 5. Edge and texture descriptors.

\begin{tabular}{lcc}
\hline \multicolumn{1}{c}{ Name } & Abbreviation & Descriptor \\
\hline Histogram of oriented Gaussians & HOG & Edge \\
Local binary pattern & LB & Texture \\
Haralick & HA & Texture \\
Zernike Moments & ZM & Shape \\
Parameter-free threshold adjacency statistics & PFTAS & Texture \\
\hline
\end{tabular}


294 Finally, the performance of Accuracy, Precision, and Recall scores were calculated according to 295 Equation 1 and shown in Tables 6 and 7.

$$
\text { Accuracy }=\frac{T P+T N}{T P+F P+F N+T N}
$$

Equation 1. Accuracy, Precision, and Recall scores calculation. TP - true positive, TN - true negative, FP-false positive, FN-false negative (according to the ground thought, see Validation 302 chapter).

$$
\text { Precision }=\frac{T P}{T P+F P}
$$$$
\text { Recall }=\frac{T P}{T P+F N}
$$

Table 6. Random Forest model for image features. Ca. 3200 objects per class. Average of 10 independent trainings.

\begin{tabular}{ccccccc}
\hline Method & Precision & SD & Recall & SD & Accuracy & SD \\
\hline HOG & 0.8493 & 0.0097 & 0.8895 & 0.0110 & 0.8634 & 0.0053 \\
LB & 0.9346 & 0.0076 & 0.9547 & 0.0077 & 0.9429 & 0.0048 \\
HA & 0.9075 & 0.0100 & 0.9216 & 0.0071 & 0.9109 & 0.0056 \\
ZM & 0.7816 & 0.0144 & 0.8239 & 0.0075 & 0.7919 & 0.0066 \\
PFTAS & 0.8821 & 0.0070 & 0.9288 & 0.0082 & 0.9000 & 0.0042 \\
\hline
\end{tabular}

Table 7. Random Forest model for image features. Ca. 10000 objects per class. Average of 10 independent trainings.

\begin{tabular}{ccccccc}
\hline Method & Precision & SD & Recall & SD & Accuracy & SD \\
\hline HOG & 0.8472 & 0.0081 & 0.8893 & 0.0080 & 0.8641 & 0.0059 \\
LB & 0.9346 & 0.0076 & 0.9547 & 0.0077 & 0.9429 & 0.0048 \\
HA & 0.9088 & 0.0057 & 0.9311 & 0.0059 & 0.9186 & 0.0046 \\
ZM & 0.6841 & 0.0116 & 0.7419 & 0.0120 & 0.7018 & 0.0064 \\
PFTAS & 0.8516 & 0.0082 & 0.8830 & 0.0055 & 0.8653 & 0.0056 \\
\hline
\end{tabular}




\section{$312 \quad$ 5.2.3 Convolutional neural network}

313 We implemented a standard convolutional neural network (Figure 3) with dropout 0.2 and trained 314 two training sets with different sizes (ca. 3200 and 10000 images per class) over 25 epochs. We 315 used rectified linear activation function during training, followed by a final SoftMax activation 316 function to receive the probability distribution over the classes. In addition, we used the stochastic 317 gradient descent optimizer with a learning rate of 0.01 , batch size of 32 , and momentum of 0.9 to 318 allow one training image to pass through the neural network at a time and update the weights for 319 each layer. The final validation accuracy of the model was 97.13\% (Figure 4).

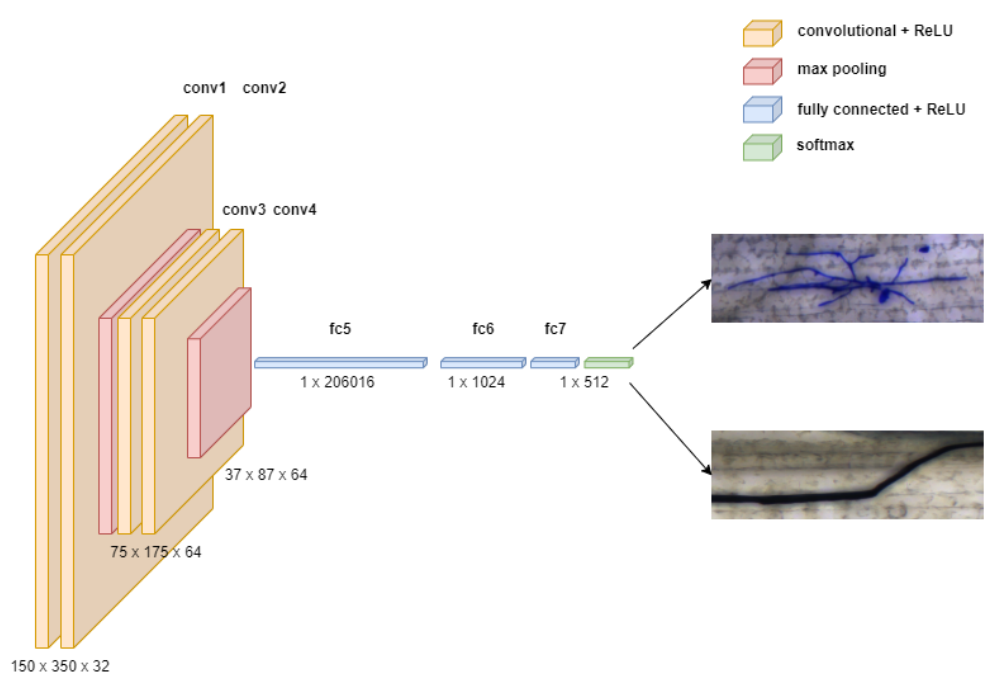

321 Figure 3. The structure of a convolutional neural network consists of convolutional, pooling, and 322 fully connected layers.
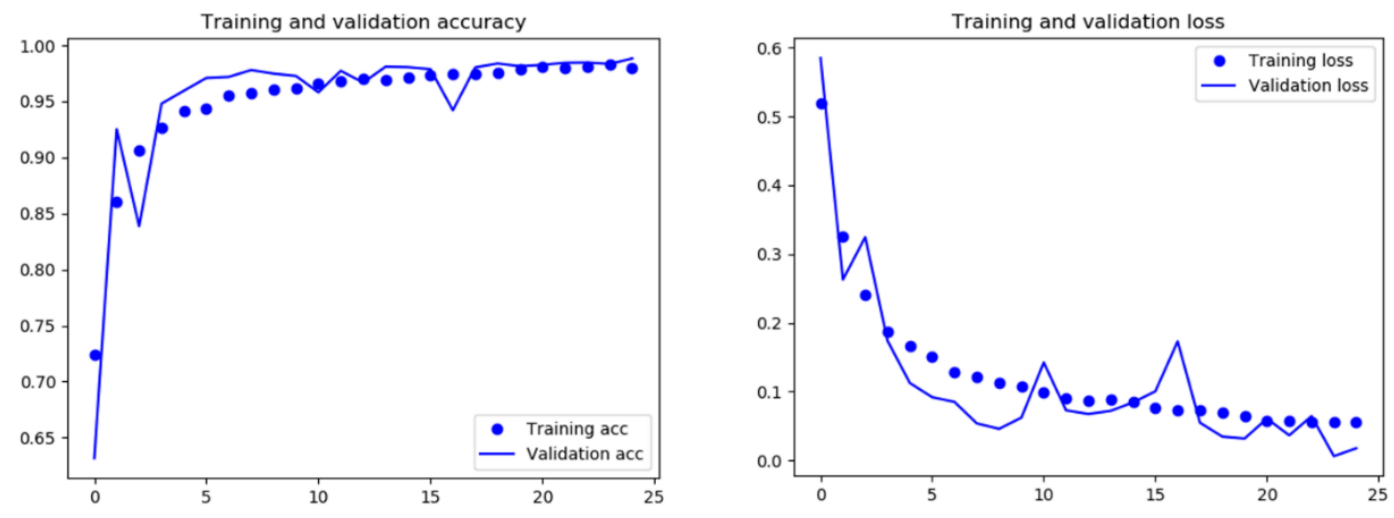

325 Figure 4. Training and validation accuracy of the model CNN model trained with ca. 10000 


\subsection{Validation}

329 One hundred twenty colonies were labeled manually as ground truth by a domain expert. When 330 comparing the handcrafted features random forest models trained on 3200 images per class, the 331 local binary pattern feature reached the highest accuracy and precision ( $>0.94$; Table 6$)$ but failed 332 when using the model on the validation set (False negative > 90\%; Table 8). This is usually an 333 indication of model overfitting resulting in a too stringent prediction or a poor capability to deal 334 with new data. This example demonstrates how misleading the theoretical performance metrics can be if used solely without validating the model with new experimental data. Re-testing all previously built models with a new validation data set revealed the Parameter-free threshold adjacency statistics (PFTAS) and haralick (HA) as best performing (True positives > $88 \%$, False positives < 10\%). Furthermore, a new model based on the combination of both methods significantly improved the accuracy ending up with $91 \%$ true positives, $9 \%$ false negatives, and only $1 \%$ false positives objects on the validation set (Table 8 ).

341 However, increasing the training data size to 10000 images did not significantly improve the 342 handcrafted feature-based model results, which indicates that the learning curve reached the 343 plateau (Table 7). In contrast, the CNN models gain from big data and larger training sets. By 344 using the dataset with 10000 images, the true positive rate increases by $3.3 \%$ to $89.1 \%$, and the 345 false-positive rate decreases to $0.0 \%$ (with the prediction accuracy score set to the maximum of 346 1.0) (Table 8). Loosening the prediction accuracy score to 0.9 helped achieve a high-performance 347 CNN model with over 98\% true positive rate and below 3\% false-positive rate. In direct 348 comparison, the CNN model shows $10 \%$ better accuracy in predicting hyphal objects than the top 349 handcrafted RF-model while keeping the false positive 7\% lower (Table 8).

350 Comparing our best CNN model with a 0.9 prediction score against the HyphArea software, our 351 proposed software improved the true positive prediction by more than $70 \%$ and decreased the false 352 positive rate by $10 \%$ (Table 8 ).

\section{$353 \quad$ 5.3.1 Run-time and parallel processing}

354 Considering the aim to do high-throughput microscopy image analysis, we invested in optimizing 355 the algorithm for run-time per image. Besides other improvements, using numerical Python 356 libraries, which allows efficient numerical calculations on multi-dimensional arrays, and 357 parallelizing the processes with the joblib library (Python) led to a significant speed gain. As a 
result, BluVision Micro performed up to 30 times faster than the previous HyphArea software in analyzing pyramid images of average size 30000 x 25000 pixels. On an Intel ${ }^{\circledR}$ Core $^{\text {TM }}$ i7-9700 CPU 3.00 GHz with 64-Bit Windows 10 operating system and NVIDIA TITAN X GPU support, the software run time takes about 60 seconds per slide containing two images of size $30000 \times 25$ 000 pixels, which is 3-5 faster than the image acquisition time, this allowing real-time analysis.

\subsubsection{Feature Visualization}

364 Visualizing the $\mathrm{CNN}$ predictions becomes crucial because of the increasing requirements for transparency of the artificial intelligence prediction models. However, the availability of visualization options was limited until recently, when several such tools were developed. To examine the BluVision Micro CNN model's prediction and facilitate debugging, we used Keras Visualization Toolkit (Zhou et al., 2015) to generate heatmap images to visualize the Class activation maps for the fungal structures. The resulting heatmaps correctly represented the area covered by the fungal microcolonies (Figure 5).

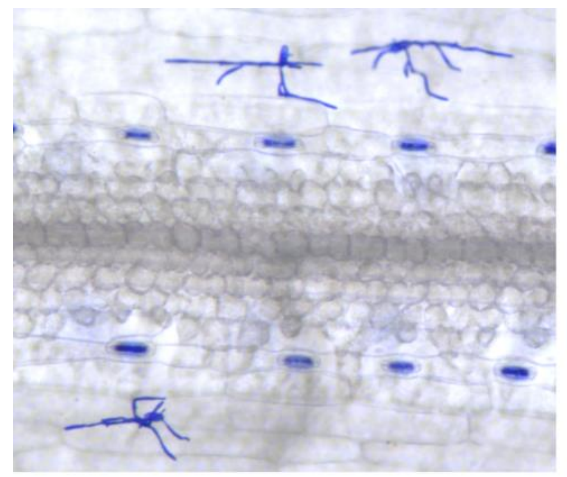

371

372

373

374

375

376

377

378

379

380

381 drops significantly (blue colors).

\subsection{Application}

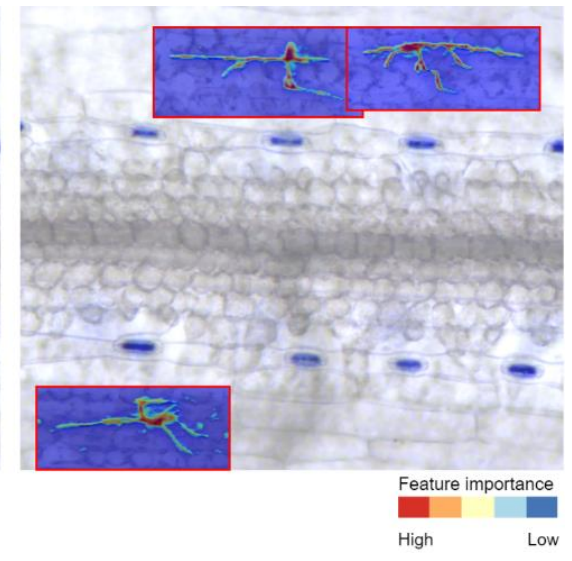

Figure 5. Heatmap visualization of the class activation map for fungal structures. The left image represents the raw image data, and on the right are the regions of interest detected by the software (red border rectangle) with hyphae segmentation. The example clearly shows that the CNN model localizes the fungal colony with high probability (red colors), as the probability in the background

\subsubsection{Genome-wide association scans (GWAS)}

The experiment design (Figure 1) allowed the quantification of multiple phenotypes (Table 1) from a single leaf. They cover the response to adapted and non-adapted pathogens on microscopic 
and macroscopic levels. The precise phenotypic data was combined with the dense SNP data (949 174 quality SNPs) for GWAS for resistance-associated markers.

Since the study aims to provide proof of concept and application examples, the number of tested genotypes was 200, which is on the lower end to detect significant marker-trait associations (MTA) in genetically diverse materials. Nevertheless, we were able to identify eight loci containing MTAs with statistical significance above the suggestive threshold $(-\log 10 \mathrm{P} \geq 6.0)$ and three loci with MTA above the significance threshold ( $-\log 10 \mathrm{P} \geq 8.0)$. Surprisingly, the novel nonhost resistance phenotypes achieved the highest association peaks leading, besides finding other MTAs, to the rediscovering one of the very few published nonhost resistance QTL (Romero et al., 2018) (Figure $6 \mathrm{E})$. All discovered significant MTAs and the genes located in the underlying genomic region are listed in Supplemental Tables MTA_list_[phenotype] and Gene_list_[phenotype].

The macroscopic phenotyping (Bgh_168hai_area) (Figure 6) suffered from some barley genotypes' apparent tendency to accelerate senescence in detached leaf assay and formation of physiological necrotic flecks that prevent the spreading of the disease and compromise the phenotyping.

Figure 6. Manhattan plot of the [- $\left.\log _{10}\right]$ transformed p-values of the genomic regions associated with the macroscopic phenotype of infected leaf area at 168 hai Bgh. Green dashed line suggestive threshold, red dashed line - significance threshold.

402 The colony size-based phenotypes (Bgh_48hai_size, Bgh_96hai_size) (Figure 7a and 7b) did not 403 deliver significant MTAs (Figure 7). This is not unexpected because a natural resistance based on 404 fungal growth retardation, to our best knowledge, is not yet described in the literature, not at last 405 because of the lack of screening methods. However, such phenotypes likely exist, and a systematic 406 screen of diverse plant genotypes may help discover them. 
a)

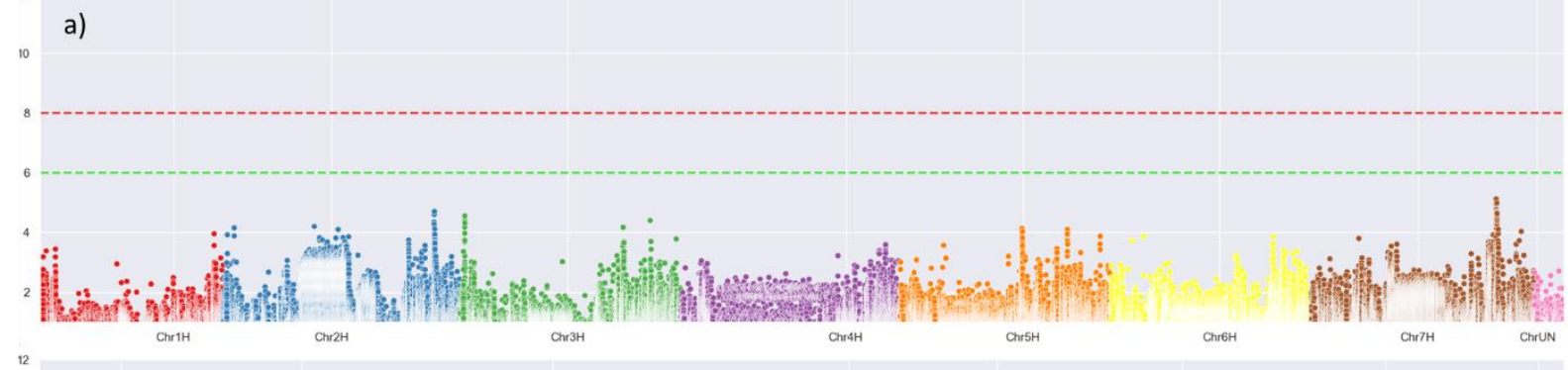

b)

Figure 7. Manhattan plot of the [- $\log _{10]}$ transformed p-values of the genomic regions associated with colony size-based phenotypes a) Bgh colony size at 48hai, b) Bgh colony size at 96hai. Green dashed line - suggestive threshold, red dashed line - significance threshold.

413 Also, as expected, the colony counts delivered some significant MTAs (Figure 8), since the 414 penetration resistance against powdery mildew fungus, which efficiently reduces the number of 415 successful infection events, is widespread in barley. However, the MTA reached only the 416 suggestive threshold, not the significance threshold, which is pretty high because of the large 417 number of SNP included in the analysis ( 1 000000$).$

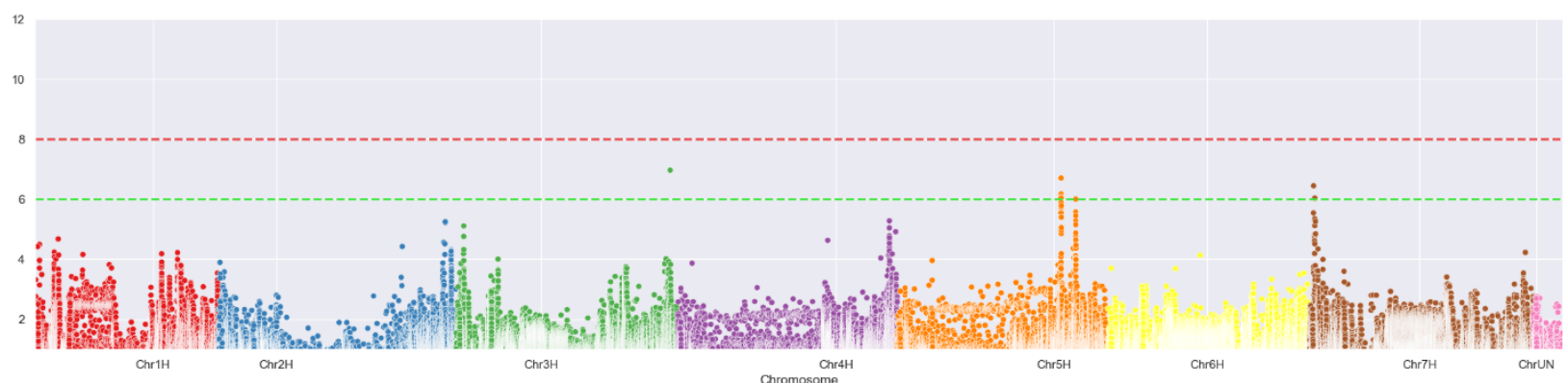

419 Figure 8 Manhattan plot of the [- $\log _{10]}$ transformed p-values of the genomic regions associated 420 with normalized Bgh colony counts at 48 hai. Green dashed line-suggestive threshold, red dashed line - significance threshold. 
423 The high sensitivity and performance of the system allowed approaching an exciting novel 424 phenotype - quantifying the rare cryptic infection of non-adapted pathogens, which allowed the 425 discovery of genes and loci associated with this most valuable type of resistance (Figure 9).
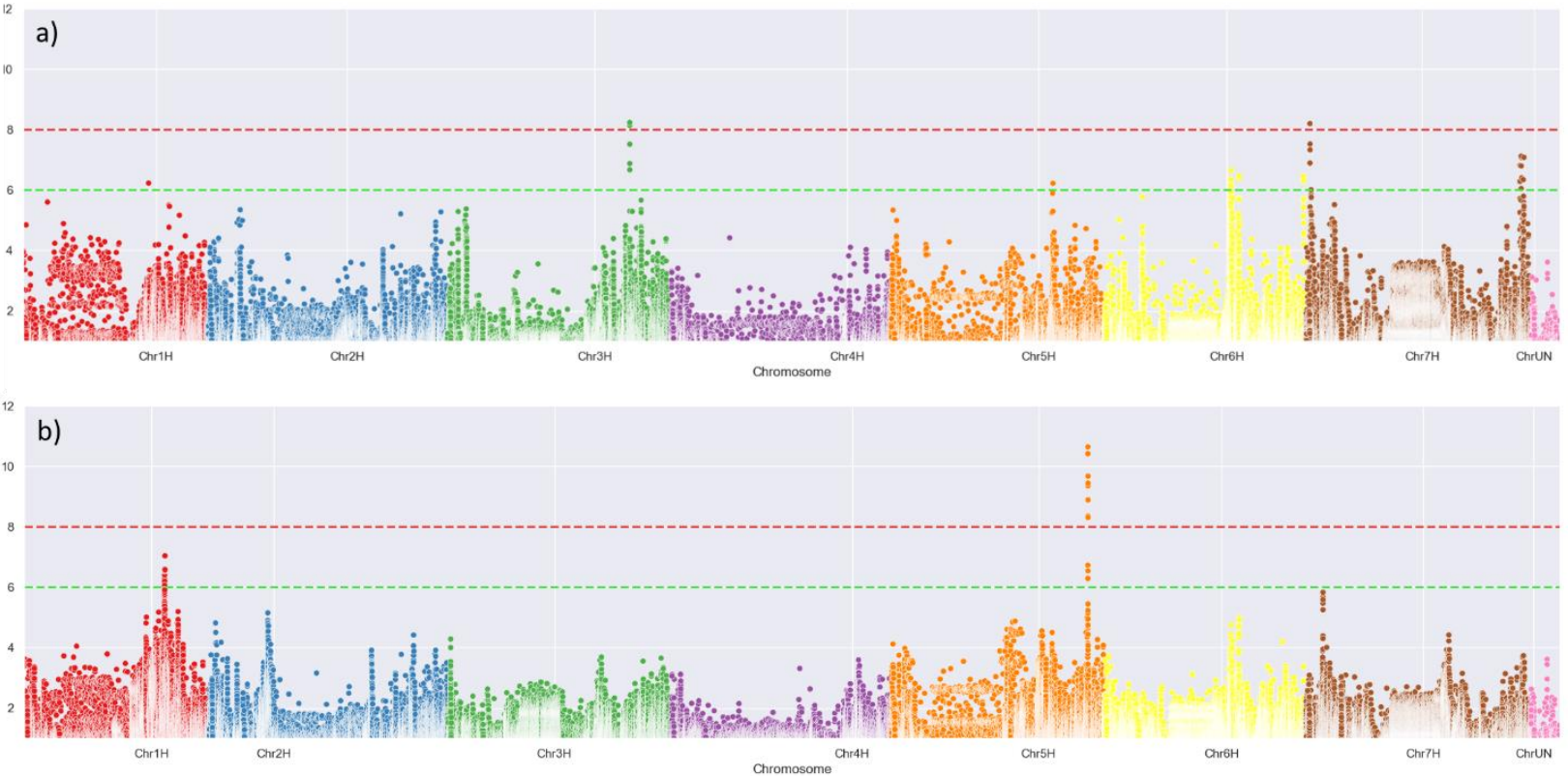

Figure 9. Manhattan plot of the [-log 10$]$ transformed p-values of the genomic regions associated with normalized Bgt colony counts (a), and binarized susceptibility phenotype (b) at 96 hai. Green dashed line - suggestive threshold, red dashed line - significance threshold.

431 Surprisingly, this novel phenotype delivered the most significant MTAs, indicating the 432 involvement of major-effect genes. Furthermore, the MTA with the absolute most significant p433 value in the entire experiment pointed precisely to the peak marker position found by (Romero et 434 al., 2018) and probably conferred by one or both of the Receptor-like kinases located in this region.

\subsubsection{Pathogen growth curves}

437 The BluVision Micro platform provides the possibility to measure precisely, and in high438 throughput, the area of the secondary hyphae of the powdery mildew colonies. This opens new 439 phenotyping options, hardly possible with the previously existing manual tools. For instance, 440 measuring the colony size at a specific time point after inoculation may reveal plant defense 441 mechanisms that rely on retarding the pathogen growth, e.g., cutting the nutrient support for the 
442 fungus or late activation of cell death mechanisms. Furthermore, acquiring colony size data on

443 multiple time points will allow for building growth curves for the pathogen (Figure 10).

444

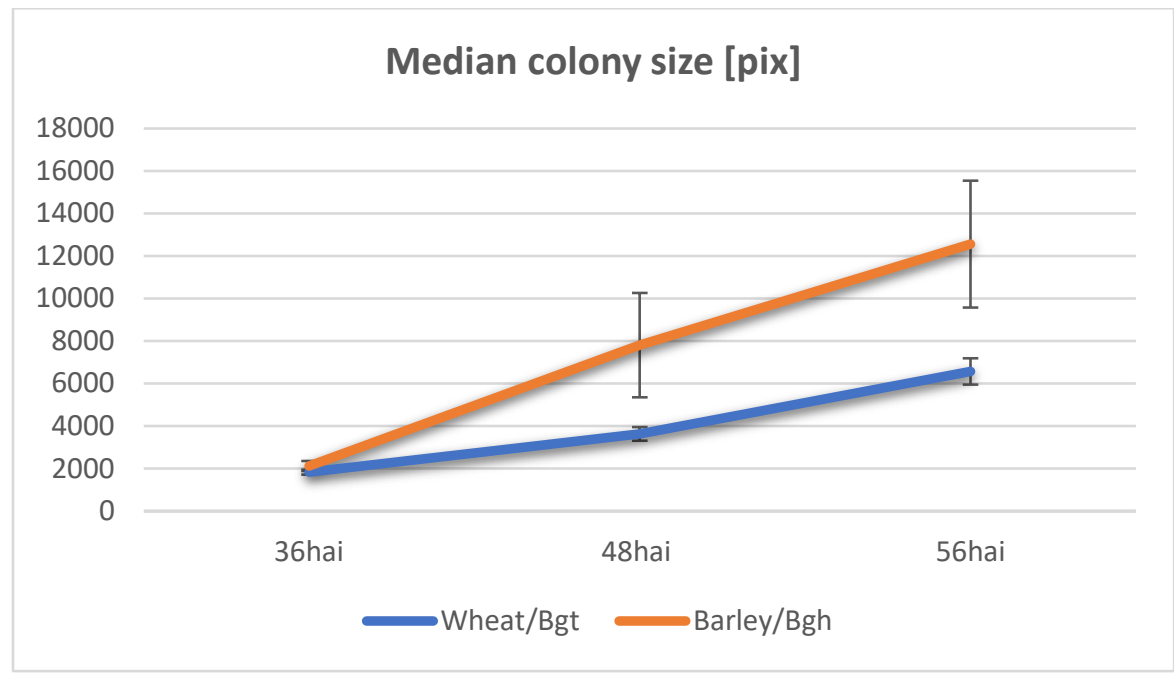

Figure 10. Growth curve of two adapted powdery mildew species on wheat and barley, respectively.

We used the median Bgh colony sizes at 48 and 96 hai on the 200 barley genotypes to build genotype-specific growth slopes and used them as a phenotype in GWAS. As for the direct colony size phenotypes, none of the MTAs reached even the suggestive threshold with the derivative one.

452 Nevertheless, this novel phenotyping method may reveal plant resistance that works by retarding 453 the pathogen growth. Also, it can be a valuable tool in comparing the fitness of different pathogen 454 races.

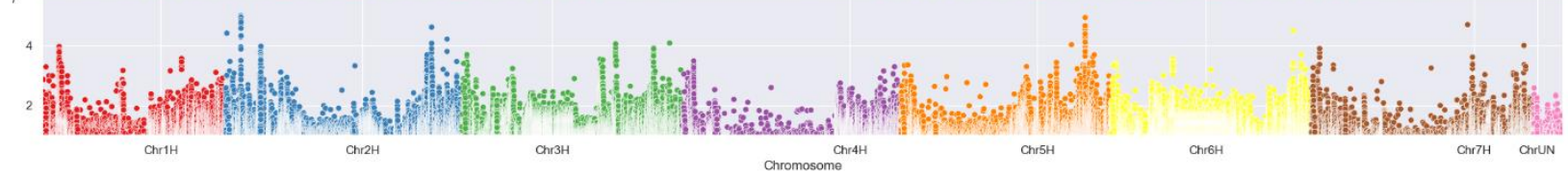

Figure 11. Manhattan plot of the [-log 10] transformed p-values of the genomic regions associated with the slope of the growth curve of Bgh at 48-96 hai. Green dashed line-suggestive threshold, red dashed line - significance threshold. 


\section{Discussion}

461 The need for automated microscopic phenotyping of plant-pathogen interactions became apparent 462 with increasing the available genetic and genomics resources and the pursuit of finding and 463 validating the functions of the myriad of genes putatively involved in the complex disease resistance phenotype. HyphArea was the first software implementation to detect and quantify secondary hyphae of $B$. graminis on barley and wheat. The tool pioneered establishing a highthroughput platform for plant-pathogen interaction phenotyping on a microscopic level allowed access to novel phenotypes such as quantification for the fungal hyphae area. However, the high sensitivity and specificity levels of the HyphArea Tool demonstrated in (Seiffert and Schweizer, 2005; Baum et al., 2011) was often difficult to reach due to differences in the material quality and variations of the sample preparations.

471 Besides the image analysis, the extended use of the HyphArea revealed issues with the handling 472 and processing of the raw data. The acquired image data were exported as individual camera 473 frames (tiles) and stored in separate TIFF files. This step simplifies the image data processing and 474 avoids using proprietary file formats but results in a massive expansion of the file number $\left(>10^{6}\right.$ 475 files for a large screen), thus approaching the limits of the used hardware and software. Finally, 476 the high run time of the HyphArea renders it less appropriate for high-throughput phenotyping 477 screenings.

478 Benefiting from the accumulated experience and using newer high-throughput automated 479 microscopy and software techniques, we have developed a completely new system for microscopy480 based phenotyping. We decided to opt for a modular, machine learning-based software that works 481 directly with different image data types, including complex pyramid files and multimodal images, 482 and it is easily adaptable and extendable with modules for additional phenotypes.

483 Handcrafted features, if chosen correctly, can provide acceptable performance in cases where only 484 small (<5 000 images per class) training sets are available. However, using more training data for 485 the handcrafted features approach does not further increase the performance, showing that we have 486 reached the methods' limits in this case. For higher accuracy and larger training sets (> 5000 487 images per class), we recommend using a CNN, which is a major advantage is extracting the 488 probability for each class and use it as a parameter for predictions. 
The BluVision system can derive precise microscopy phenotypes for different large-scale studies, such as screening of Genebank material, crossing populations, mutant collections, breeding material, and others, at both host and pathogen sides. In this study, we have used the system to screen 200 highly genetically diverse barley genotypes for interaction phenotypes with adapted and non-adapted powdery mildew fungi. The system was confirmed to deliver accurate, sensitive, and reproducible results. We have used them to scan for marker-trait associations in the barley genome, discover several novel loci, and confirm already known. Noticeably, we were able to rediscover one of the first published nonhost-resistance QTL, described by (Romero et al., 2018), which confirms the system's applicability for studies aiming to discover genes involved in this precious but hardly accessible trait - the nonhost resistance. Furthermore, the systems allow highthroughput studies of previously extremely laborious phenotypes, such as precise colony area and scoring pre- and post-haustorial defense reactions. By using other (not yet published) dedicated modules, the BluVision platform can also detect the presence of fungal haustoria in reporter gene (GUS) expressing cells, thus enabling high-throughput transfection assays for disease resistancerelated genes. The open-source software system allows the development of specific modules for other microscopic phenotypes. The framework is hardware-independent and adaptable to different commercial imaging systems based on the Digital Imaging and Communications in Medicine (DICOM) standard, such as Zeiss Axionscan and Leica Aperio systems.

Thus, we have developed an open-source, extendable, high-throughput automated microscopy system for analyzing microscopic phenotypes. Furthermore, we have validated the system's performance in disease resistance screens of genetically diverse barley material and demonstrated that the phenotypic data could be used for Genome-wide associations scans (GWAS), discovering several resistance-associated loci, including conferring nonhost resistance.

\section{Acknowledgments}

514 This work was initiated and actively supported by Patrick Schweizer, who passed away in March

515 2018. The authors dedicate this work to his memory. Further on, we would like to acknowledge 516 the following colleagues from IPK Gatersleben: Nils Stein, Martin Marcher, Murukarthick 517 Jayakodi for the genotypic data for the 200 barley accessions; Andreas Börner for providing single518 seed-descent seed material for the experiments; and André Fessel and Gabriele Brantin for the 
519 technical help. The work was supported by IPK Gatersleben and the German Ministry of Education

520 and Research (BMBF) with grants FKZ 031A053 (DPPN) and FKZ 031B0184 (GeneBank 2.0).

\section{Author Contribution}

522 SL and DD wrote the manuscript, SL performed the image analysis, computer model development, 523 and GWAS, DD designed the research and performed the biological experiments.

524

5259 Data Availability

526 BluVision Micro ships with Attribution-NonCommercial 4.0 International license (CC BY-NC

527 4.0). The open-source code is accessible at https://github.com/snowformatics/BluVisionMicro.

528 Image training sets are available at the electronic Data Archive Library (e!DAL) (link with the 529 next manuscript revision). 


\section{References}

Baum T, Navarro-Quezada A, Knogge W, Douchkov D, Schweizer P, Seiffert U (2011) HyphArea-Automated analysis of spatiotemporal fungal patterns. Journal of Plant Physiology 168: 72-78

Bettgenhaeuser J, Hernandez-Pinzon I, Dawson AM, Gardiner M, Green P, Taylor J, Smoker M, Ferguson JN, Emmrich P, Hubbard A, Bayles R, Waugh R, Steffenson BJ, Wulff BBH, Dreiseitl A, Ward ER, Moscou MJ (2021) The barley immune receptor Mla recognizes multiple pathogens and contributes to host range dynamics. Nature Communications 12

Bourras S, Kunz L, Xue MF, Praz CR, Muller MC, Kalin C, Schlafli M, Ackermann P, Fluckiger S, Parlange F, Menardo F, Schaefer LK, Ben-David R, Roffler S, Oberhaensli S, Widrig V, Lindner S, Isaksson J, Wicker T, Yu DZ, Keller B (2019) The AvrPm3-Pm3 effector-NLR interactions control both race-specific resistance and host-specificity of cereal mildews on wheat. Nature Communications $\mathbf{1 0}$

Canny J (1986) A computational approach to edge detection. IEEE Trans Pattern Anal Mach Intell 8: $679-698$

Coelho LP, Ahmed A, Arnold A, Kangas J, Sheikh A-S, Xing EP, Cohen WW, Murphy RF (2010) Structured Literature Image Finder: Extracting Information from Text and Images in Biomedical Literature. In. Springer Berlin Heidelberg, Berlin, Heidelberg, pp 23-32

Dalal N, Triggs B (2005) Histograms of Oriented Gradients for Human Detection. In Proceedings of the 2005 IEEE Computer Society Conference on Computer Vision and Pattern Recognition (CVPR'05) - Volume 1 - Volume 01. IEEE Computer Society, pp 886-893

De K, Masilamani V (2013) Image Sharpness Measure for Blurred Images in Frequency Domain. Procedia Engineering 64: 149-158

Dong-chen H, Li W (1990) Texture Unit, Texture Spectrum, And Texture Analysis. IEEE Transactions on Geoscience and Remote Sensing 28: 509-512

Douchkov D, Luck S, Johrde A, Nowara D, Himmelbach A, Rajaraman J, Stein N, Sharma R, Kilian B, Schweizer P (2014) Discovery of genes affecting resistance of barley to adapted and non-adapted powdery mildew fungi. Genome Biology $\mathbf{1 5}$

FAO (2020) FAO launches 2020 as the UN's International Year of Plant Health. In, Vol 2020. FAI, http://www.fao.org/news/story/en/item/1253551/icode/

Glasbey CA (1993) An Analysis of Histogram-Based Thresholding Algorithms. CVGIP: Graphical Models and Image Processing 55: 532-537

Haralick RM, Shanmugam K, Dinstein I (1973) Textural Features for Image Classification. Ieee Transactions on Systems Man and Cybernetics Smc3: 610-621

Hayabuchi Y, Inoue M, Watanabe N, Sakata M, Nabo MMH, Kagami S (2011) Minimumintensity projection of multidetector-row computed tomography for assessment of pulmonary hypertension in children with congenital heart disease. International Journal of Cardiology 149: 192-198 
Henderson CR (1975) Best Linear Unbiased Estimation and Prediction under a Selection Model. Biometrics 31: 423-447

Hinton G (1999) Unsupervised Learning: Foundations of Neural Computation. In. The MIT Press

Hommel G (1988) A Stagewise Rejective Multiple Test Procedure Based on a Modified Bonferroni Test. Biometrika 75: 383-386

Khamfongkhruea C, Thongsawad S, Tannanonta C, Chamchod S (2017) Comparison of CT images with average intensity projection, free breathing, and mid-ventilation for dose calculation in lung cancer. Journal of applied clinical medical physics 18: 26-36

König P, Beier S, Basterrechea M, Schüler D, Arend D, Mascher M, Stein N, Scholz U, Lange M (2020) BRIDGE - A Visual Analytics Web Tool for Barley Genebank Genomics. Frontiers in Plant Science 11

Li CH, Lee CK (1993) Minimum cross entropy thresholding. Pattern Recognition 26: 617-625

Li CH, Tam PKS (1998) An iterative algorithm for minimum cross entropy thresholding. Pattern Recognition Letters 19: 771-776

Lin W, Hasenstab K, Moura Cunha G, Schwartzman A (2020) Comparison of handcrafted features and convolutional neural networks for liver MR image adequacy assessment. Scientific Reports 10: 20336

Lippert C, Listgarten J, Liu Y, Kadie CM, Davidson RI, Heckerman D (2011) FaST linear mixed models for genome-wide association studies. Nature Methods 8: 833-835

Listgarten J, Lippert C, Kadie CM, Davidson RI, Eskin E, Heckerman D (2012) Improved linear mixed models for genome-wide association studies. Nature Methods 9: 525-526

Liu X-Q, Rong J-Y, Liu X-Y (2008) Best linear unbiased prediction for linear combinations in general mixed linear models. Journal of Multivariate Analysis 99: 1503-1517

Lück S, Strickert M, Lorbeer M, Melchert F, Backhaus A, Kilias D, Seiffert U, Douchkov D (2020) "Macrobot": An Automated Segmentation-Based System for Powdery Mildew Disease Quantification. Plant Phenomics 2020: 5839856

Lueck S, Beukert U, Douchkov D (2020) BluVision Macro - a software for automated powdery mildew and rust disease quantification on detached leaves. The Journal of Open Source Software 5(51)

Mascher M (2020) Assembly, annotation and analysis of the barley (Hordeum vulgare L.) pangenome. In. e!DAL - Plant Genomics and Phenomics Research Data Repository (PGP), IPK Gatersleben, Seeland OT Gatersleben, Corrensstraße 3, 06466, Germany

Milner SG, Jost M, Taketa S, Mazón ER, Himmelbach A, Oppermann M, Weise S, Knüpffer H, Basterrechea M, König P, Schüler D, Sharma R, Pasam RK, Rutten T, Guo G, Xu D, Zhang J, Herren G, Müller T, Krattinger SG, Keller B, Jiang Y, González MY, Zhao Y, Habekuß A, Färber S, Ordon F, Lange M, Börner A, Graner A, Reif JC, Scholz U, Mascher M, Stein N (2019) Genebank genomics highlights the diversity of a global barley collection. Nature Genetics 51: 319-326

Mitchell TM (1997) Machine Learning, 
Otsu N (1979) A Threshold Selection Method from Gray-Level Histograms. IEEE Transactions on Systems, Man, and Cybernetics 9: 62-66

Panstruga R, Dodds PN (2009) Terrific Protein Traffic: The Mystery of Effector Protein Delivery by Filamentous Plant Pathogens. Science 324: 748-750

Stockman G, Shapiro LG (2001) Computer Vision. Prentice Hall PTR

Tahmasbi A, Saki F, Shokouhi SB (2011) Classification of benign and malignant masses based on Zernike moments. Comput. Biol. Med. 41: 726-735

Voulodimos A, Doulamis N, Doulamis A, Protopapadakis E (2018) Deep Learning for Computer Vision: A Brief Review. Computational Intelligence and Neuroscience 2018: 7068349

Wang L, He D-C (1990) Texture classification using texture spectrum. Pattern Recognition 23: 905-910

Weisstein EW (2021) Moore Neighborhood In MathWorld - A Wolfram Web Resource, Vol 2021, MathWorld - A Wolfram Web Resource. https://mathworld.wolfram.com/MooreNeighborhood.html

Yang W, Guo Z, Huang C, Duan L, Chen G, Jiang N, Fang W, Feng H, Xie W, Lian X, Wang G, Luo Q, Zhang Q, Liu Q, Xiong L (2014) Combining high-throughput phenotyping and genome-wide association studies to reveal natural genetic variation in rice. Nature Communications 5: 5087

Yen JC, Chang FJ, Chang S (1995) A new criterion for automatic multilevel thresholding. IEEE Trans Image Process 4: 370-378 
650 Zack GW, Rogers WE, Latt SA (1977) Automatic measurement of sister chromatid exchange 651 frequency. J Histochem Cytochem 25: 741-753

652 Zheng A, Casari A (2018) Feature Engineering for Machine Learning: Principles and Techniques 653 for Data Scientists. O'Reilly Media, Inc.

654 Zhou B, Khosla A, Lapedriza A, Oliva A, Torralba A (2015) Learning Deep Features for 655 Discriminative Localization, 